\title{
Involvement of the Wnt signaling pathway in feeder-free culture of human induced pluripotent stem cells
}

\author{
MINORU TOMIZAWA ${ }^{1}$, FUMINOBU SHINOZAKI ${ }^{2}$, YASUFUMI MOTOYOSHI ${ }^{3}$, \\ TAKAO SUGIYAMA $^{4}$, SHIGENORI YAMAMOTO ${ }^{5}$ and NAOKI ISHIGE ${ }^{6}$ \\ Departments of ${ }^{1}$ Gastroenterology, ${ }^{2}$ Radiology, ${ }^{3}$ Neurology, ${ }^{4}$ Rheumatology, ${ }^{5}$ Pediatrics and ${ }^{6}$ Neurosurgery, \\ National Hospital Organization, Shimoshizu Hospital, Yotsukaido, Chiba 284-0003, Japan
}

Received November 22, 2014; Accepted August 25, 2015

DOI: $10.3892 / \mathrm{mmr} .2015 .4314$

\begin{abstract}
Activin A maintains the pluripotency of human induced pluripotent stem (hiPS) cells. A combination of activin A and CHIR99021 (CHIR), a specific inhibitor of glycogen synthase- $3 \beta$, is suitable for feeder-free culture of hiPS cells. In the present study, the specific role of the Wnt signaling pathway in cells cultured under different conditions was investigated. Following transfection with the reporter plasmids, TOPflash and FOPflash, hiPS cells were cultured in medium, containing activin A, CHIR, leukemia inhibitory factor (LIF) or SB431542, a specific inhibitor of activin A. A luciferase reporter assay was performed $48 \mathrm{~h}$ later. Western blot analysis was performed to determine the expression levels of $\beta$-catenin and tubulin- $\alpha$. The activity of Wnt in hiPS cells was suppressed by culture in the presence of activin A. The activation of the Wnt pathway was most marked when the cells were cultured with a combination of activin A and CHIR. Addition of SB431542 into the culture revealed no significant change in the Wnt pathway. Western blot analysis revealed that $\beta$-catenin accumulated most often in cells cultured with activin A and CHIR. $\beta$-catenin also accumulated in cells cultured with activin A alone. Culture with activin A and CHIR most effectively stimulated the Wnt signaling pathway, as measured by luciferase assays using TOPflash and FOP flash as reporter plasmids. $\beta$-catenin accumulated in the hiPS cells cultured with activin $\mathrm{A}$, via a mechanism, which remains to be elucidated. The Wnt signaling pathway may be important for hiPS cell growth in feeder-free culture.
\end{abstract}

\section{Introduction}

Human induced pluripotent stem (hiPS) cells are a promising cell source for regenerative medicine. To avoid exposure

Correspondence to: Dr Minoru Tomizawa, Department of Gastroenterology, National Hospital Organization, Shimoshizu Hospital, 934-5 Shikawatashi, Yotsukaido, Chiba 284-0003, Japan E-mail: nihminor-cib@umin.ac.jp

Key words: activin A, luciferase activity, $\beta$-catenin, CHIR99021, SB431542 of hiPS cells to proteins from species other than human, feeder-free culture conditions have been developed (1). Several factors have been identified, which are part of the signaling network of hiPS cells (2). Leukemia inhibitory factor (LIF) is involved in the pluripotency of mouse embryonic stem cells (3). In human embryonic stem (hES) cells, Nodal/activin A is involved in pluripotency (3). Activin A maintains the pluripotency of hiPS cells under feeder-free conditions by regulating the expression of Nanog (4-6). Basic fibroblast growth factor (bFGF) sustains the self-renewal capacity of hES cells (7). CHIR99021 (CHIR), a glycogen synthase kinase (GSK)-3 $\beta$ inhibitor, improves the ability of hiPS cells to maintain pluripotency by acting synergistically with activin A (8). The development of hiPS cells from human embryonic keratinocytes via the activation of Oct $3 / 4$ and Klf4 was reported to be driven by culture with CHIR (9). Mouse iPS cells can also be generated in culture medium containing Oct3/4 and CHIR (10). Finally, mouse embryonic stem cells maintain pluripotency when cultured with CHIR (11). Taken together, these reports suggest that CHIR is involved in the maintenance of pluripotency. Since GSK-3 $\beta$ is involved in the activation of the Wnt signaling pathway, it is likely that the Wnt pathway is important in pluripotency (12).

The Wnt signaling pathway is mediated by Disheveled (Dsh) proteins in Xenopus. Dsh proteins inhibit GSK-3 $\beta$ and promote the accumulation of $\beta$-catenin. Dsh homolog (Dvl)2, a mammalian homolog of Dsh, is phosphorylated upon stimulation by the Wnt signaling pathway and mediates endocytosis of the frizzled receptor $(13,14)$. Once GSK-3 $\beta$ has been inactivated via phosphorylation of Ser9 by Dvl2, $\beta$-catenin accumulates in the cytoplasm and subsequently translocates into the nucleus (15).

The role of the Wnt signaling pathway in hiPS cells is controversial. The Wnt signaling pathway has been demonstrated to be involved in pluripotency (16) and accumulation of $\beta$-catenin by inhibition of GSK-3 $\beta$ sustains pluripotency in conjunction with other factors (17). Indeed, the addition of the GSK-3 $\beta$ inhibitor to the culture facilitates the efficient generation of hiPS cells (18). On the other hand, the Wnt signaling pathway has been demonstrated to promote the differentiation of hiPS cells in conjunction with other factors. For instance, stage-specific activation of bone morphogenetic protein and 
the Wnt signaling pathway promotes the differentiation of iPS cells into epicardial cells (19). The combination of the Wnt signaling pathway and bFGF promotes the differentiation of iPS cells into nephron progenitor cells (20). Therefore, the present study investigated the direct role of the Wnt signaling pathway in maintaining hiPS pluripotency under feeder-free culture conditions (8).

\section{Materials and methods}

Cell culture. The hiPS cells (201B7; Riken Cell Bank, Tsukuba, Japan) were cultured in 6-well plates (Asahi Techno Glass, Tokyo, Japan) coated with Matrigel ${ }^{\mathrm{TM}}$ (Becton Dickinson, Franklin Lakes, NJ, USA) in ReproFF medium (Reprocell, Yokohama, Japan) for feeder-free culture in $5 \% \mathrm{CO}_{2}$ at $37^{\circ} \mathrm{C}$ in a humidified chamber. The cells were harvested using Accutase $^{\circledR}$ (Innovative Cell Technologies, Inc., San Diego, CA, USA) for experiments. A total of $10 \mathrm{ng} / \mathrm{ml}$ activin A (R\&D Systems, Inc., Minneapolis, MN, USA), $2 \mu$ M GSK-3 $\beta$ inhibitor (CHIR; Wako Pure Chemicals Industries, Ltd., Osaka, Japan), 1,000 U/ml human LIF (Sigma-Aldrich, St. Louis, MO, USA) or the SB431542 activin A inhibitor (Wako Pure Chemicals Industries, Ltd.) was added to Dulbecco's Minimum Essential Medium-F12 (Sigma-Aldrich), supplemented with $20 \%$ Knockout Serum Replacement (Life Technologies, Grand Island, NY, USA), 10\% Minimum Essential Amino Acids (Life Technologies), 2 mM 1-Glutamine (Life Technologies), and $1 \mathrm{mM}$ 2-Mercaptoethanol [iPSm(-); Sigma-Aldrich].

Luciferase assay. The 201B7 cells were seeded into 24-well plates, coated with Matrigel ${ }^{\mathrm{TM}}$, and cultured for $24 \mathrm{~h}$. Once the cells reached $70 \%$ confluence, they were transfected with Lipofectamine LTX (Life Science Technologies), and $0.5 \mu \mathrm{g}$ TOPflash reporter plasmid or FOPflash reporter plasmid (EMD Millipore, Temecula, CA, USA) and $0.05 \mu \mathrm{g}$ pRL-TK (Promega, Madison, WI, USA) was added to the medium. The transcriptional activity was measured using a dual luciferase reporter assay system (Promega) using Gene Light (GL-200A; Microtech Co. Ltd., Funabashi, Japan). Non-transfected cells were used as a negative control. The ratio of the luciferase activity of TOPflash against that of FOPflash was calculated. The relative Wnt activity was determined by normalizing this ratio against that of $201 \mathrm{~B} 7$ cells cultured in ReproFF medium.

Western blot analysis. The total protein was isolated from the cells following culture for $48 \mathrm{~h}$. A $10 \mu \mathrm{g}$ protein sample was separated on $12 \%$ sodium dodecyl sulfate (Wako Pure Chemicals Industries, Ltd.)-polyacrylamide (Bio-Rad, Hercules, CA, USA) gel electrophoresis gels and was subsequently transferred to a nylon filter (EMD Millipore). Following blocking for $30 \mathrm{~min}$ with $5 \%$ non-fat milk, the filters were incubated with rabbit monoclonal anti-human $\beta$-catenin antibodies (1:2,500; cat. no. \#9582; Cell Signaling Technology, Inc., Danvers, MA, USA) for $1 \mathrm{~h}$ at room temperature. The filters were washed in phosphate-buffered saline (PBS) three times, and subsequently incubated with horseradish peroxidase (HRP)-conjugated anti-rabbit antibody (1:2,500; cat. no. NA934-100UL; GE Healthcare Life

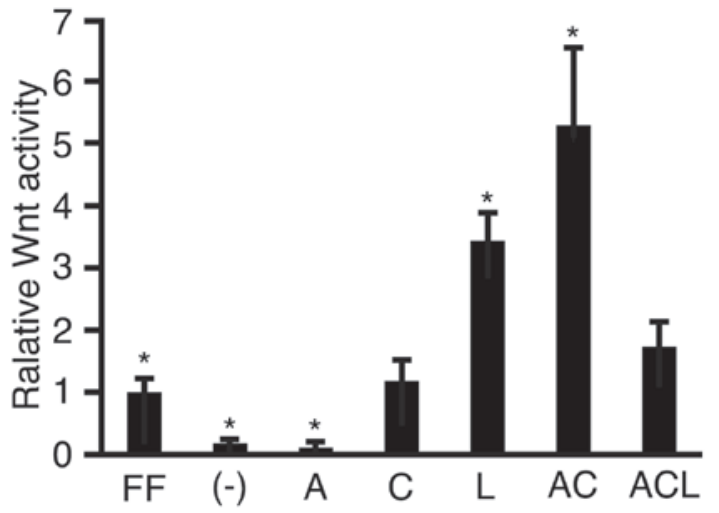

Figure 1. Luciferase reporter assay. Human induced pluripotent stem cells were transfected with the reporter plasmids, TOPflash or FOPflash, cultured in various media and subjected to a luciferase reporter assay. The ratios of TOPflash/FOPflash in the transfected cells were normalized against the cells cultured with ReproFF alone, and the resulting value was expressed as the relative Wnt activity. The data are presented as the mean \pm standard deviation ( $\mathrm{P}<0.05 ; \mathrm{n}=3)$. FF, ReproFF; (-), iPSm(-); A, activin A; C, CHIR99021; L, leukemia inhibitory factor; AC, activin A + CHIR99021; ACL, activin A, CHIR99021 + leukemia inhibitory factor.

Sciences, Little Chalfont, UK) at room temperature for $1 \mathrm{~h}$. After three washes with PBS, the specific antigen-antibody complexes were visualized using enhanced chemiluminescence (cat. no. RPN2232; GE Healthcare Life Sciences). The filter was reprobed with 10 Minute Western Re-probe kit (cat. no. JZ-008; Jacksun Easy Biotech Inc., New York, NY, USA), and incubated with mouse monoclonal anti-human $\alpha$-tubulin antibody (cat. no. MS-581-P0; 1:2,500; Lab Vision Corporation, Fremont, CA, USA) for $1 \mathrm{~h}$ to use as a loading control. The filters were washed with PBS, and incubated with HRP-linked anti-mouse antibody (cat. no. NA931100UL; GE Healthcare Life Sciences) for $1 \mathrm{~h}$. The specific antigen-antibody complexes were visualized using enhanced chemiluminescence (cat. no. RPN2232; GE Healthcare Life Sciences) after three washes with PBS. The expression levels of $\beta$-catenin were normalized against that of $\alpha$-tubulin and were analyzed using ImageJ 64 imaging software (National Institutes of Health, Bethesda, MD, USA).

Statistical analysis. Statistical analysis was performed by one-factor analysis of variance using JMP 10.0.2 (SAS Institute, Cary, NC, USA). $\mathrm{P}<0.05$ was considered to indicate a statistically significant difference.

\section{Results}

To investigate activity of the Wnt signaling pathway, a luciferase assay was performed using TOPflash and FOPflash as reporter plasmids (Fig. 1). Following transfection, activin A, CHIR and/or LIF was added to the cell culture medium. The relative Wnt activity was lower in the cells cultured in iPSm(-) $(0.18 \pm 0.05 ; \mathrm{P}<0.05)$ compared with the ReproFF cultured cells. The addition of activin A suppressed the activity of Wnt when compared with the cells cultured in iPSm(-) alone $(0.11 \pm 0.05)$. By contrast, the Wnt signaling pathway was markedly activated in cells cultured with activin A and CHIR (5.28 \pm 0.23 ; $\mathrm{P}<0.05)$. These results suggested that the combination of 
Lane $\begin{array}{lllll}1 & 2 & 3 & 4 & 5\end{array}$

\section{$\begin{array}{lllll}1.00 & 0.94 & 1.80 & 1.90 & 2.47\end{array}$}

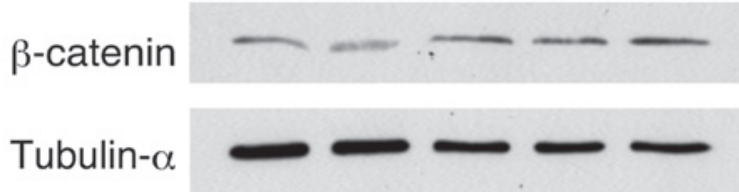

Figure 2. Western blot analysis to determine the expression of $\beta$-catenin. The expression levels of $\beta$-catenin and tubulin- $\alpha$ were analyzed by western blotting. The signal intensity of $\beta$-catenin was divided by that of tubulin- $\alpha$ to generate the relative signal intensity, as indicated above the band lanes. The cell treatment types in each lane were as follows: 1, ReproFF; 2, iPSm(-); 3 , activin A; 4, activin A + CHIR99021; 5, activin A, CHIR99021 + leukemia inhibitory factor.

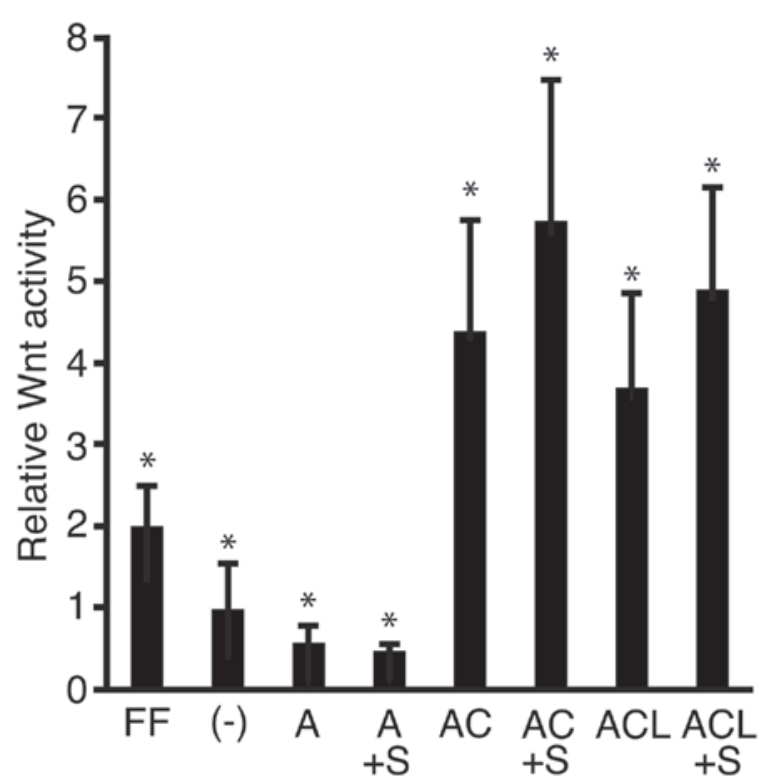

Figure 3. Luciferase reporter assay. Human induced pluripotent stem cells transfected with the reporter plasmids, TOPflash or FOPflash, were cultured in various media and were subjected to a luciferase reporter assay. The ratios of TOPflash/FOPflash in transfected cells were normalized against the cells cultured with ReproFF alone and the resulting value was expressed as the relative Wnt activity. The data are presented as the mean \pm standard deviation ( $\mathrm{P}<0.05 ; \mathrm{n}=3$ ). FF, ReproFF; (-), iPSm(-); A, activin A; A + S, activin A + SB431542; AC, activin A + CHIR99021; ACS, activin A, CHIR99021 + SB431542; ACL, activin A, CHIR99021 + leukemia inhibitory factor; ACLS, activin A, CHIR99021, leukemia inhibitory factor + SB431542

activin A and CHIR in the culture medium clearly stimulated the Wnt pathway.

To analyze the accumulation of $\beta$-catenin, western blot analysis was performed following the addition of activin A, CHIR and/or LIF to the culture medium (Fig. 2). The signal intensity of $\beta$-catenin was divided by the intensity of tubulin- $\alpha$ to obtain the relative signal intensity. The relative signal intensity of $\beta$-catenin detected in cells cultured in iPSm(-) (0.94) was lower compared with that of the cells cultured in ReproFF. The relative signal intensity of $\beta$-catenin detected in the cells cultured in medium with activin A (1.80), activin A + CHIR (1.90) or the combination of activin A, CHIR + LIF (2.47) was higher compared with that detected in the cells cultured in iPSm(-) alone. These results suggested that $\beta$-catenin accumulated in response to culture with activin A and other factors.

To address the possibility that activin A stimulated the Wnt signaling pathway to a comparable level of ReproFF and SB431542, a specific inhibitor of activin A was added to the culture medium, and a luciferase assay was performed (Fig. 3). The relative Wnt activity was lower in the cells cultured in media containing activin A $(0.28 \pm 0.11)$ compared with those cultured in $\mathrm{PSSm}(-)(0.49 \pm 0.27)$. The relative Wnt activity of the cells cultured in medium with activin A + CHIR (2.20 \pm 0.68 ; $\mathrm{P}<0.05)$ and the combination of activin A, CHIR + LIF $(1.85 \pm 0.58 ; \mathrm{P}<0.05)$ was higher compared with that in the cells cultured in ReproFF alone. These results were consistent with those shown in Fig. 1. The relative Wnt activity was lower in the cells cultured with activin A + SB431542 compared with those cultured in the presence of activin A alone. Addition of SB431542 to the culture marginally increased the relative Wnt activity simulated by culture with activin $\mathrm{A}+\mathrm{CHIR}$, and the combination of activin A, CHIR + LIF. However, these differences were non-significant.

\section{Discussion}

Activin A is essential for the maintenance of the pluripotency of hiPS cells cultured under feeder-free conditions $(4,21)$. In our previous study, hiPS cells were cultured under feeder-free conditions in the presence of activin A and CHIR (8). Since CHIR is involved in activation of the Wnt signaling pathway, it was hypothesized that activin A and the Wnt signaling pathway cooperate to maintain the pluripotency and proliferation of iPS cells. In the present study, the relative Wnt activity and the expression of $\beta$-catenin were investigated. As expected, the relative Wnt activity was greatest in the cells cultured with a combination of activin A and CHIR. These results indicated that the activation of the Wnt signaling pathway was optimal for the feeder-free culture of iPS cells.

Unexpectedly, the cells cultured in medium, supplemented with only activin A, resulted in the accumulation of $\beta$-catenin. It was unclear why $\beta$-catenin accumulated in the culture supplemented with activin A, while the relative Wnt activity was suppressed. These results may represent crosstalk between activin A and the Wnt signaling pathway (22).

In the present study, one of the limitations was that the expression levels of GSK $3 \beta$ and Dvl2 remained to be determined. The expression levels of GSK3 $\beta$ and Dvl2 has been previously demonstrated to increase when the Wnt signaling pathway is stimulated (23). An analysis of these molecules is required to fully elucidate the mechanism underlying the activation of the Wnt signaling pathway, and future studies in our laboratory will focus on this line of research.

In conclusion, culture with medium containing activin A and CHIR stimulated the Wnt signaling pathway, as measured using a luciferase assay with the TOPflash and FOP flash reporter plasmids. The addition of activin A to the culture medium was associated with $\beta$-catenin accumulation, although the underlying mechanism remains to be elucidated. Overall, these results suggested that the Wnt signaling pathway may be required for the maintenance of pluripotency in hiPS cells cultured under feeder-free conditions. 


\section{References}

1. Meng G, Liu S and Rancourt DE: Synergistic effect of medium, matrix and exogenous factors on the adhesion and growth of human pluripotent stem cells under defined, xeno-free conditions. Stem Cells Dev 21: 2036-2048, 2012.

2. Dalton S: Signaling networks in human pluripotent stem cells. Curr Opin Cell Biol 25: 241-246, 2013.

3. Dowell KG, Simons AK, Bai H, Kell B, Wang ZZ, Yun K and Hibbs MA: Novel insights into embryonic stem cell self-renewal revealed through comparative human and mouse systems biology networks. Stem Cells 32: 1161-1172, 2014.

4. Tomizawa M, Shinozaki F, Sugiyama T, Yamamoto S, Sueishi M and Yoshida T: Activin A maintains pluripotency markers and proliferative potential of human induced pluripotent stem cells. Exp Ther Med 2: 405-408, 2011.

5. Shin M, Alev C, Wu Y, Nagai H and Sheng G: Activin/TGF-beta signaling regulates Nanog expression in the epiblast during gastrulation. Mech Dev 128: 268-278, 2011.

6. Miyanari Y and Torres-Padilla ME: Control of ground-state pluripotency by allelic regulation of Nanog. Nature 483: 470-473, 2012.

7. Xu RH, Peck RM, Li DS, Feng X, Ludwig T and Thomson JA: Basic FGF and suppression of BMP signaling sustain undifferentiated proliferation of human ES cells. Nat Methods 2: 185-190, 2005.

8. Tomizawa M, Shinozaki F, Sugiyama T, Yamamoto S, Sueishi M and Yoshida T: Activin A is essential for Feeder-free culture of human induced pluripotent stem cells. J Cell Biochem 114 584-588, 2013.

9. Li W, Zhou H, Abujarour R, Zhu S, Young Joo J, Lin T, Hao E, Schöler HR, Hayek A and Ding S: Generation of human-induced pluripotent stem cells in the absence of exogenous Sox2. Stem Cells 27: 2992-3000, 2009.

10. Li Y, Zhang Q, Yin X, Yang W, Du Y, Hou P, Ge J, Liu C, Zhang W, Zhang X, et al: Generation of iPSCs from mouse fibroblasts with a single gene, Oct 4 and small molecules. Cell Res 21: 196-204, 2011.

11. Ying QL, Wray J,Nichols J, Batlle-Morera L, Doble B, Woodgett J, Cohen P and Smith A: The ground state of embryonic stem cell self-renewal. Nature 453: 519-523, 2008.
12. Wu D and Pan W: GSK3: A multifaceted kinase in Wnt signaling. Trends Biochem Sci 35: 161-168, 2010.

13. Itoh K, Brott BK, Bae GU, Ratcliffe MJ and Sokol SY: Nuclear localization is required for Dishevelled function in Wnt/beta-catenin signaling. J Biol 4: 3, 2005.

14. Chen W,ten BergeD,BrownJ,AhnS,HuLA, MillerWE, CaronMG, Barak LS, Nusse R and Lefkowitz RJ: Dishevelled 2 recruits beta-arrestin 2 to mediate Wnt5A-stimulated endocytosis of Frizzled 4. Science 301: 1391-1394, 2003.

15. McCubrey JA, Steelman LS, Bertrand FE, Davis NM, Sokolosky M, Abrams SL, Montalto G, D'Assoro AB, Libra M, Nicoletti F, et al: GSK-3 as potential target for therapeutic intervention in cancer. Oncotarget 5: 2881-2911, 2014.

16. Holland JD, Klaus A, Garratt AN and Birchmeier W: Wnt signaling in stem and cancer stem cells. Curr Opin Cell Biol 25: 254-264, 2013.

17. Sineva GS and Pospelov VA: $\beta$-Catenin in pluripotency: Adhering to self-renewal or Wnting to differentiate? Int Rev Cell Mol Biol 312: 53-78, 2014.

18. Bar-Nur O, Brumbaugh J, Verheul C, Apostolou E, Pruteanu-Malinici I, Walsh RM, Ramaswamy $S$ and Hochedlinger K: Small molecules facilitate rapid and synchronous iPSC generation. Nat Methods 11: 1170-1176, 2014.

19. Witty AD, Mihic A, Tam RY, Fisher SA, Mikryukov A, Shoichet MS, Li RK, Kattman SJ and Keller G: Generation of the epicardial lineage from human pluripotent stem cells. Nat Biotechnol 32: 1026-1035, 2014.

20. Lam AQ, Freedman BS and Bonventre JV: Directed differentiation of pluripotent stem cells to kidney cells. Semin Nephrol 34: 445-461, 2014.

21. Pauklin S and Vallier L: The cell-cycle state of stem cells determines cell fate propensity. Cell 155: 135-147, 2013.

22. Singh AM, Reynolds D, Cliff T, Ohtsuka S, Mattheyses AL, Sun Y, Menendez L, Kulik M and Dalton S: Signaling network crosstalk in human pluripotent cells: A Smad2/3-regulated switch that controls the balance between self-renewal and differentiation. Cell Stem Cell 10: 312-326, 2012.

23. Tomizawa M, Shinozaki F, Motoyoshi Y, Sugiyama T, Yamamoto S, Sueishi M and Yoshida T: Niclosamide suppresses Hepatoma cell proliferation via the Wnt pathway. Onco Targets Ther 6: 1685-1693, 2013. 